The Economic Journal, 120 (June), 595-611. doi: 10.1111/j.1468-0297.2009.02312.x. (C) The Author(s). Journal compilation (C) Royal Economic Society 2009. Published by Blackwell Publishing, 9600 Garsington Road, Oxford OX4 2DQ, UK and 350 Main Street, Malden, MA 02148, USA.

\title{
THE FOURFOLD PATTERN OF RISK ATTITUDES IN CHOICE AND PRICING TASKS*
}

\author{
William T. Harbaugh, Kate Krause and Lise Vesterlund
}

\begin{abstract}
We examine the robustness of the fourfold pattern of risk attitudes under two elicitation procedures. We find that individuals are, on average, risk-seeking over low-probability gains and high-probability losses and risk-averse over high-probability gains and low-probability losses when we elicit prices for the gambles. However, a choice-based elicitation procedure, where participants choose between a gamble and its expected value, yields individual decisions that are indistinguishable from random choice. Sensitivity to elicitation procedure holds between and within participants, and remains when participants are allowed to review and change decisions. The price elicitation procedure is more complex; this finding may be further evidence that an increase in cognitive load exacerbates behavioural anomalies.
\end{abstract}

Individual decisions over risky outcomes often deviate from that predicted by expected utility theory, and alternative models have been proposed to explain behaviour better. ${ }^{1}$ Perhaps the most accepted alternative is cumulative prospect theory (CPT) by Tversky and Kahneman (1992). ${ }^{2}$ Two central assumptions in CPT are that individuals are riskaverse over gains and risk-seeking over losses, and that they tend to overweight lowprobability events while underweighting the likelihood of high-probability ones. Combined these two assumptions may result in a unique pattern of risk attitudes. As stated by Tversky and Kahneman (1992): 'The most distinctive implication of prospect theory is the fourfold pattern of risk attitudes'. ${ }^{3}$ Specifically, it is predicted that when faced with a risky prospect people will be:

(1) risk-seeking over low-probability gains,

(2) risk-averse over high-probability gains,

(3) risk-averse over low-probability losses and

(4) risk-seeking over high-probability losses.

The objective of this article is to examine the robustness of the fourfold pattern using two different elicitation procedures. We asked 128 people to evaluate a small set of simple gambles with low and high probabilities of cash gains and losses. In one price-based procedure we use the Becker-DeGroot-Marschak (BDM) procedure to

* This research was funded by grants from the National Science Foundation and the Preferences Network of the MacArthur Foundation. We thank Mary Ewers, Aaron Kaminsky and Irana Abibova for help running the experiments. We also thank Colin Camerer, Stefano DellaVigna, John Duffy, Drew Fudenberg, Ed Glaeser, David Laibson, George Loewenstein, Muriel Niederle, Matthew Rabin, Al Roth, Stefan Trautmann and seminar participants at Berkeley, Case Western, CMU, Cornell, Harvard, Syracuse and UCLA for helpful comments and conversations.

${ }^{1}$ For reviews of the literature see for example, Schoemaker (1982), Machina (1987) and Starmer (2000). For examples of comparisons between the alternative models, see e.g., Harless and Camerer (1994) Hey and Orme (1994).

2 Cumulative prospect theory is a generalisation of prospect theory, Kahneman and Tversky (1979). Camerer (1998) argues that cumulative prospect theory is supported by the preponderance of evidence and he suggests that it is time to abandon expected utility theory in its favour. Camerer (2000) makes a similar recommendation.

${ }^{3}$ Tversky and Kahneman (1992), p. 306. 
elicit participants' willingness to pay for the lotteries and, in the other choice-based procedure, we ask them to choose between the gamble and its expected value. This allows us to observe whether individuals make decisions that are consistent with each of the four elements of the fourfold pattern and whether those decisions are affected by the elicitation procedure.

We find that the fourfold pattern is a very good predictor of risk attitudes - but only when people are asked to report their willingness to pay for a risky prospect. When they are instead asked to choose between the gamble and its expected value, we find that their decisions are not distinguishable from random choice. This result holds both between and within participants and does not depend on the ordering of tasks. We also show that the change in elicited preferences between the two methods remains even after participants review their price and choice responses simultaneously and are allowed to change them.

There are several potential explanations for the sensitivity to the elicitation procedure. One such explanation may be found in the literature on dual selves. ${ }^{4}$ The dual-self models argue that cognitive load may decrease an individual's ability to exert willpower over the more impulsive self. Thus an increase in cognitive load may result in more substantial behavioural anomalies. Interestingly a recent experimental study by Benjamin et al. (2006) shows that cognitive load increases both small-stakes riskaversion and short-run discounting. To the extent that the cognitive load of the BDM price procedure is greater than in the choice-based procedure our finding may be seen as further evidence that cognitive load exacerbates behavioural anomalies.

In Section 1 of the article we review the literature that motivates our study. Section 2 presents our experimental design. Sections $3 \& 4$ show how the results support the conclusion that the fourfold pattern is present in pricing tasks but not in choice tasks. Section 5 discusses various explanations for the sensitivity to elicitation procedure and concludes the article.

\section{Motivation}

As mentioned in the Introduction, CPT's assumptions on the value and probability weighting functions give rise to the fourfold pattern of risk attitudes. First, CPT assumes that preferences can be described by a reference-dependent value function $v(x)$, where $x$ denotes the change in the payoff from a person's initial wealth position. As shown in Figure 1(a), the value function is kinked at the endowment point with a steeper slope for losses than gains, thus capturing the loss aversion finding that losses loom larger than gains. Furthermore, based on evidence that people are risk-averse over gains and risk-seeking over losses, the value function is assumed to be concave for gains and convex for losses. Second, rather than responding to the objective probability $\mathrm{p}$, it is assumed that individuals weight these by a non-linear probability weighting function $w(\mathrm{p})$, as illustrated in Figure $1(b)$. Impossible events are discarded such that $w(0)=0$ and the scale is normalised such

\footnotetext{
${ }^{4}$ See e.g., Bernheim and Rangel (2004), Loewenstein and O'Donoghue (2005), Brocas and Carillo (2005), Fudenberg and Levine (2006) and Ozdenoren et al. (2006).

(C) The Author(s). Journal compilation (C) Royal Economic Society 2009
} 
(a)

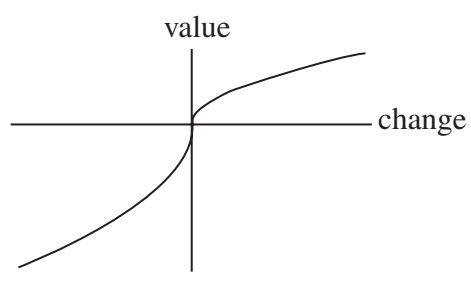

(b) subjective probability

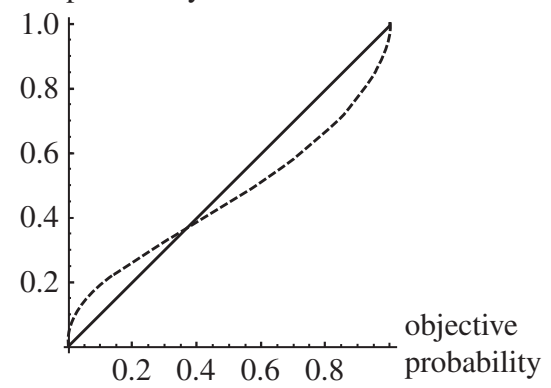

Fig. 1. The Prospect Theory Value and Weighting Functions,

(a) The Value Function, (b) The Subjective Probability Weighting Function

that $w(1)=1 .^{5}$ To capture the finding that individuals are insensitive to changes in the probability, the weighting function is assumed to be 'regressive' and, as shown in Figure $1(b)$, to cut the diagonal from above. Thus people are assumed to overweight low probability events and underweight high probability ones. Kahneman and Tversky's predicted fourfold pattern results when the magnitude of $w(\mathrm{p})$ is large relative to $v(x)$. That is, the overweighting of low-probabilities needs to be large enough that people are risk-seeking for lotteries with low-probability gains and riskaverse for low-probability losses. Note however that probability weighting alone will give rise to the fourfold pattern when individuals are risk neutral.

While there is a large experimental literature on decision making under risk very few studies directly test the fourfold pattern of risk attitudes. The focus of much of the literature is on testing CPT against alternative models by relying on choices over a large set of relatively complex gambles. For example, Hey and Orme (1994) show people 100 pairs of (mostly) three-outcome lotteries and have them choose one of each pair, or report indifference. The lotteries are fairly complicated - for example, individuals chose between a lottery with 0.375 chance of $£ 10,0.125$ chance of $£ 20$ and 0.5 chance of $£ 30$, and one with a 0.125 chance of $£ 10,0.750$ chance of $£ 20$ and 0.125 chance of $£ 30$. ${ }^{6}$ Other studies focus on estimating the shape of the value function, the weighting function, or both. Gonzalez and Wu (1999) use an iterative procedure to elicit certainty equivalents for 300 two-outcome gambles, all over gains, with varying probabilities and payoffs. Participants are paid $\$ 50$ for completing the four-hour-long task and, in

\footnotetext{
${ }^{5}$ Kahneman and Tversky (1979) present a probability weighting function that is discontinuous at the bounds to demonstrate that the function is not well-behaved on the boundary. Subsequent presentations, including those of Tversky and Kahneman (1992), are nonetheless of a continuous probability weighting function. We too illustrate a continuous probability function to demonstrate the characteristics of the continuous functions estimated by Camerer and Ho (1994), Gonzalez and Wu (1999), Prelec (1998), Tversky and Kahneman (1992), Tversky and Fox (1995), Wu and Gonzalez (1996).

6 The odds are shown with pie charts on a computer and one randomly chosen decision is played for real money. Hey and Orme (1994) report that neither of several varieties of probability weighting models provide better explanations of the data than does expected utility theory. They repeat the experiment a week later and find many differences in choices, suggesting that decision errors are an important aspect of decision making in these experiments. See also, Harless and Camerer (1994) for analysis of 23 datasets consisting of choices over similarly complex gambles.
} 
addition, at least one participant is paid for at least one decision. The data help them to estimate both the probability weighting and the value functions and they report that results from ten of the eleven participants, who were psychology graduate students, are consistent with the CPT predictions about the shape of these functions over gains.

Direct tests of the fourfold pattern using real and simple gambles are scarce. The data for Kahneman and Tversky's 1979 paper came from survey questions about choices between an array of lotteries with large hypothetical gains and losses. Tversky and Kahneman (1992) presented 25 graduate students with a series of simple lotteries over smaller but still hypothetical, losses and gains. They used an iterative procedure to obtain close bounds on the certainty equivalents for the lotteries and found strong support for the fourfold pattern. ${ }^{7}$ However behaviour elicited from survey-type data need not mirror that over real cash lotteries (Harless and Camerer, 1994; Camerer and Hogarth, 1999; Battalio et al., 1990). More recently Holt and Laury (2002) conduct a direct comparison of decisions for hypothetical and real lotteries over gains and find that people appear more risk-seeking when faced with hypothetical rather than real gambles. Laury and Holt (2008) present the same hypothetical/real comparison for decisions over gains and over losses, and find that while the hypothetical decisions are risk-averse over gains and risk-seeking over losses, with real gambles they are risk-averse for gains but risk-neutral for losses. As the Holt-Laury procedure relies on choices between pairs of lotteries with different probabilities and expected values, it does not enable a comparison of behaviour between low and high-probability lotteries and, as a result their papers do not shed light on the full fourfold pattern.

Others have allowed for high and low-probability gambles, but focus solely on the gain domain. For example, Kachelmeier and Shehata (1992) presented Chinese, Canadian and American participants with a sequence of 25 simple real lotteries over gains and asked what price they would be willing to accept in return for their lottery ticket. They used the demand-revealing Becker-DeGroot-Marschak procedure to elicit prices. The results show substantial risk-seeking for low-probability prospects but do not show risk-aversion for high-probability prospects. In a follow-up experiment on a limited set of gambles they find evidence that the lack of risk-aversion is due to the willingness-to-accept format of their elicitation. Reported willingness to pay for a prospect is much lower than the reported willingness to accept.

Harbaugh et al. (2002) is to our knowledge unique in using real and simple gambles to directly test the full fourfold pattern. They examine decisions over a small set of simple lotteries with cash payoffs, over gains and losses and with a range of probabilities. The participants range in age from 5 to 64 . To make the protocol transparent for the youngest participants they use a very simple choice-based elicitation procedure, asking people to choose between a risky prospect with one non-zero outcome, and its expected value. They find that children's risk attitudes diverge from the fourfold pattern and, while the divergence diminishes with age, they do not find adults behaving in a manner consistent with the fourfold pattern. Our objective in this article is to determine why Harbaugh et al. (2002) in contrast to previous studies do not find evidence of the fourfold pattern. As previous evidence for the fourfold pattern has

\footnotetext{
7 Thus the elicitation method used by Tversky and Kahneman (1992) effectively asks participants to determine their willingness to pay for a hypothetical gamble.

(C) The Author(s). Journal compilation (C) Royal Economic Society 2009
} 
been observed by eliciting prices of a gamble, it may be that failure to observe the fourfold pattern is due to their choice-based elicitation procedure. Another possible explanation is that the unusual subject pool caused the results to diverge from the fourfold pattern. To address both of these explanations we determine if the fourfold pattern is robust to the elicitation procedure when using a standard subject pool.

\section{Experimental Design}

We design an experiment to test the fourfold pattern of risk attitudes using simple lotteries for cash gains and losses over a range of probabilities. Risk attitudes are elicited using both choice and price-based procedures. The choice procedure simply asks individuals to choose between a lottery and its expected value. The price procedure asks participants to report the most they are willing to pay to play a lottery over gains, or the most they are willing to pay to avoid playing a lottery over losses. ${ }^{8}$ The $\mathrm{BDM}$ procedure is used to determine whether participants will pay a randomly determined price to play the lottery (gain), or to avoid the lottery (loss). We explain the BDM procedure separately for losses and for gains. Each explanation includes an example, a test of understanding, and then a further discussion.

Participants in the experiment were asked to evaluate the six prospects shown in Table 1. Prior estimates of the probability weighting function report, and Figure 1(b) illustrates, that the absolute difference between the weighted probability and the objective probability is largest when the objective probability is 0.1 and 0.8 , and that the functions cross at approximately $0.4 .^{9}$ Therefore we are particularly interested in determining risk attitudes for prospects 1,3,4 and 6, as we expect strong support for the fourfold pattern at these prospects. The participants were college students from a variety of majors at the University of New Mexico. To allow for both between and within-participants analyses, everyone evaluated the prospects using both procedures. Sixty-four students used the choice method first and 32 used the price method first. We elected to have a larger subject pool for the choice method since this version is less common when examining the fourfold pattern. After the first elicitation procedure each group then evaluated the gambles using the other method. ${ }^{10}$ We refer to participants who first complete the choice method as 'choice-participants' and those who first complete the price method as 'price-participants'.

\footnotetext{
${ }^{8}$ With this procedure the willingness-to-pay and the choice decisions are slightly different over gains. Because people must pay to play the gambles, their payoff is reduced by the random price drawn. We address this issue in Section 5. Note that the willingness-to-accept format presents a similar difference over losses where participants accept a payment in return for the gamble. We use the willingness-to-pay format to limit the 'overbidding' that is frequently found with willingness-to-accept questions. When eliciting the monetary equivalent of a gamble one must elicit either a willingness-to-accept or willingness-to-pay measure. Schmidt et al. (2005) develop a reference-dependent model that they call third-generation prospect theory. This model predicts preference reversals between choice and willingness-to-accept (WTA) evaluation tasks because loss aversion causes a decision-maker to require greater compensation to forego a potential gain. This perceived loss is not present in the choice task. As a result, a WTA preference elicitation mechanism will lead to higher valuations of a lottery than a choice task.

${ }^{9}$ See for example Camerer and Ho (1994), Gonzalez and Wu (1999), Prelec (1998), Tversky and Kahneman (1992), Tversky and Fox (1995), Wu and Gonzalez (1996).

${ }^{10}$ Note that the participants were unaware that they would be asked to evaluate the prospects more than once.
}

(C) The Author(s). Journal compilation (C) Royal Economic Society 2009 
Table 1

The Six Prospects

\begin{tabular}{lcccc}
\hline \hline Prospect Number & Probability & Payoff & Expected Value & $\begin{array}{c}\text { Predicted FFP of } \\
\text { Risk Attitude }\end{array}$ \\
\hline 1 & 0.1 & $+\$ 20$ & $\$ 2$ & Seeking \\
2 & 0.4 & $+\$ 20$ & $\$ 8$ & Neutral \\
3 & 0.8 & $+\$ 20$ & $\$ 16$ & Averse \\
4 & 0.1 & $-\$ 20$ & $-\$ 2$ & Averse \\
5 & 0.4 & $-\$ 20$ & $-\$ 8$ & Neutral \\
6 & 0.8 & $-\$ 20$ & $-\$ 16$ & Seeking \\
\hline \hline
\end{tabular}

Each experimental session lasted about 30 minutes and only one participant at a time was present. Upon arriving at the laboratory the student was directed to a partition, where he or she could make decisions without being observed by the experimenter. Participants were randomly assigned to be either a price or a choice participant. After reading the instructions for the initial elicitation method, participants were shown a sample prospect and a spinner card of the sort used in board games. ${ }^{11}$ They were told they would be asked to make six decisions and that one decision would be picked randomly to count for their payoff. ${ }^{12}$ We then counted out $\$ 22$ in single dollar bills, put it on the table in front of them and asked them to evaluate the six prospects, one at a time. ${ }^{13}$ The odds for the gambles were shown both numerically and using spinner cards and these same spinners were used by the experimenter to determine outcomes. ${ }^{14} \mathrm{We}$ refer to the initial decision as the first-round decision. At the time the first-round decision was made the participant had no reason to believe that it was not his or her final decision. After completing the initial evaluation of one set of six decisions, the participants were then asked to lay all their decisions out on a table so that they could see them simultaneously. At this point they were given an opportunity to change any of their responses. ${ }^{15}$ We refer to decisions at this point as the second-round decisions.

We used a restart procedure to obtain decisions for the second elicitation procedure. After completing the second-round decisions of the first task, participants were asked to participate in another experiment, before their earnings from the first task were determined. Using a self-contained set of instructions, they were presented with the second elicitation method. They were given another $\$ 22$, completed the six evaluations,

11 Instructions for the experiment are posted at http://www.pitt.edu/ vester/FFPInstructions.pdf

${ }^{12}$ A similar procedure is also used by Tversky and Kahneman (1986), Starmer et al. (1998) and Camerer (1989). When offering participants to change their decision once it has been randomly selected, Camerer (1989) finds that they do not use that option. Laury (2005) shows that the procedure of randomly choosing one of several gambles elicits roughly the same preferences as when participants are paid for all of the decisions they make.

${ }^{13}$ Prospect theory will not predict the fourfold pattern unless people view this $\$ 22$ as 'theirs'. This might not be the case if people see this $\$ 22$ as a windfall gain, rather than as compensation for the time involved in participating in the experiment. In Section 2 we show that, in the pricing task, people exhibit the fourfold pattern as well as loss aversion. We take this as evidence that they do treat this payment as part of their endowment. Note also that this is the procedure that has previously been used to elicit risk attitudes over losses, see for example, Camerer (1989) and Battalio et al. (1990).

${ }^{14}$ Hertwig et al. (2004) find that individuals overweight low-probability events in decisions from description, while they underweight such events in decisions from experience. While Hertwig et al. classify decisions in our experiment as being from description it is possible that prior experience with spinners lead individuals to underweight low-probability events.

${ }^{15}$ We thank Dale Stahl for suggesting this revision procedure.

(C) The Author(s). Journal compilation (C) Royal Economic Society 2009 
and were again asked to review the six decisions simultaneously and make any changes they wished. Once both elicitation methods were completed, participants reviewed all twelve decisions simultaneously and were given a third and final opportunity to change their answers. After completing the third-round decisions, we picked one prospect from each elicitation method, played any gambles, and paid the participants their net earnings in cash, which averaged $\$ 44$ and ranged between $\$ 4$ and $\$ 84$. People did not know they would participate in the second task, nor that they would be allowed to re-evaluate their choices, so the first-round decisions allow for a clean betweenparticipants comparison of price and choice behaviour. The opportunity for the revisions was included to reduce errors but our general results are the same regardless of the round.

For each elicitation method we presented the prospects according to one of four different orders. An equal proportion of participants was given each order. Two orderings presented the prospects in increasing order of probability (from $10 \%$ to $80 \%$ ), with one ordering presenting gains first and then losses and the other ordering presenting losses first and then gains. Two other orderings presented the prospects in decreasing order of probability (from $80 \%$ to $10 \%$ ), once again one ordering first presented gains and the other first presented losses. Participants received the prospects in the same order for both the choice and pricing methods, and the order in which a person was shown the choices was determined randomly. We find that decisions do not differ significantly across these orders.

\section{Risk Attitudes from Price Elicitations}

We start by examining the risk attitudes that result when using the price elicitations. We first present the results for the price-participants who were asked to evaluate the prospects first using the price elicitation. Table 2 reports their first-round average and median prices. A participant is classified as risk-neutral if the reported price equals the expected value of the gamble. If the participant is willing to pay more than the expected value to play the gamble over gains then she is classified as risk-seeking.

Table 2

Price-participants in the Price Task

\begin{tabular}{|c|c|c|c|c|c|c|c|c|}
\hline \multicolumn{3}{|l|}{ Prospect } & \multicolumn{3}{|c|}{ Mean Reported Price } & \multicolumn{3}{|c|}{ Median Reported Price } \\
\hline Description & & $\begin{array}{l}\text { Expected } \\
\text { Value }\end{array}$ & Price & $\begin{array}{c}\text { p-value, } \\
\text { Wilcoxon Test }\end{array}$ & $\begin{array}{l}\text { Mean Risk } \\
\text { Attitude }\end{array}$ & Price & $\begin{array}{l}\text { p-value, } \\
\text { Sign Test }\end{array}$ & $\begin{array}{c}\text { Median Risk } \\
\text { Attitude }\end{array}$ \\
\hline \multirow[t]{3}{*}{ Gain $+\$ 20$} & 1. $\mathrm{p}=0.1$ & $\$ 2$ & $\$ 4.9$ & 0.007 & Seeking & $\$ 2.0$ & 0.078 & Neutral \\
\hline & 2. $\mathrm{p}=0.4$ & $\$ 8$ & $\$ 8.1$ & 0.500 & Neutral & $\$ 7.0$ & 0.170 & Averse \\
\hline & 3. $\mathrm{p}=0.8$ & $\$ 16$ & $\$ 12.2$ & 0.000 & Averse & $\$ 12.0$ & 0.000 & Averse \\
\hline \multirow[t]{3}{*}{ Loss $-\$ 20$} & 4. $\mathrm{p}=0.1$ & $-\$ 2$ & $-\$ 5.7$ & 0.000 & Averse & $-\$ 4.5$ & 0.000 & Averse \\
\hline & 5. $\mathrm{p}=0.4$ & $-\$ 8$ & $-\$ 9.6$ & 0.021 & Averse & $-\$ 9.0$ & 0.064 & Averse \\
\hline & 6. $p=0.8$ & $-\$ 16$ & $-\$ 12.6$ & 0.000 & Seeking & $-\$ 13.0$ & 0.000 & Seeking \\
\hline
\end{tabular}

Notes. 32 participants, first-round decisions. The Wilcoxon test assumes the price distribution is symmetric and tests the hypothesis that the mean and median of the distribution equal the expected value of the gamble. The sign test does not assume symmetry and tests the hypothesis that the median of the distribution equals the expected value of the gamble.

(C) The Author(s). Journal compilation (C) Royal Economic Society 2009 
Similarly, she is classified as risk-seeking if the amount she is willing to pay to avoid playing a gamble involving a loss is less than the expected value.

We first note that the prices reported for the low and high-probability prospects differ substantially from the associated expected values. ${ }^{16}$ Second, consistent with CPT's assumption of loss aversion we see that losses loom larger than similar sized gains. Both the mean and median prices for a positive prospect are smaller than the absolute value of the prices reported for the corresponding negative prospect. ${ }^{17}$ Third, the mean reported prices imply risk attitudes that are consistent with the fourfold pattern. When presented with a prospect involving a gain participants are risk-seeking at low-probability gains and risk-averse at high-probability ones. Over losses, risk attitudes reflect and we see the opposite pattern. In all four cases the risk attitude implied by mean prices is significantly different from risk-neutrality. This pattern is also supported by the median prices. The only exception is prospect 1 , where the median price equals the expected value of the gamble. Thus, across participants the price elicitation results in risk attitudes that are very much in line with the fourfold pattern.

A similar result holds within participants, where we directly can assess the individual reflections in risk attitudes when moving from low to high probabilities of winning, or when moving from the gain to the loss domain. Conditional on the stake of the prospect being a loss or a gain, the first panel of Figure 2 shows the proportion of participants whose reported prices suggest that they are risk-averse versus risk-seeking for the high and low-probability prospects (High $\mathrm{P}$ and Low $\mathrm{P}$, respectively). The second panel shows the proportion with each combination of risk attitudes when conditioning on the likelihood of the stake and recording risk attitudes for prospects with a similar sized loss and gain. The highlighted cells are the outcomes predicted by prospect theory.

The within-participant support for the fourfold pattern of risk attitudes is striking. The modal cell in the price task is always consistent with the predicted reflection of risk attitudes and, in two of the four cases, more than half the participants are in the predicted cell. Risk attitudes reflect in two dimensions: conditional on a gain or a loss, attitudes reflect when moving from a high to a low-probability prospect; conditional on a low or a high-probability prospect, attitudes reflect between a gain and a loss.

We determine statistically whether the proportion with the predicted risk attitudes exceeds the proportion that would be expected if participants were equally likely to have any combination of risk attitudes. With three different risk attitudes and hence 9 possible combinations we use an exact binomial test of proportions to test the null that at most $1 / 9$ are in the cell predicted by the fourfold pattern of risk attitudes. ${ }^{18}$ In all four comparisons we can reject the null in favour of the alternative that more people are in the predicted cell, with p-values less than 0.001 . The same conclusion is reached when we exclude those who are risk-neutral and test the hypothesis that at most $25 \%$ of

\footnotetext{
16 The prices found by Tversky and Kahneman (1992) also differ substantially from the expected value. For example they find a median reported price of $\$ 9$ for a $10 \%$ chance of winning $\$ 50$.

17 Note however that only in the comparison of prospect 2 and 5 can we reject the hypothesis that the absolute price reported for a loss equals that of the similar sized gain ( $\mathrm{p}$-value of the Wilcoxon test equals $0.03)$.

18 Given the size of the gambles it may be argued that the majority of participants should be risk-neutral, thus the null distribution is not obvious. We therefore consider outcomes when including and excluding risk-neutral participants.
}

(C) The Author(s). Journal compilation (C) Royal Economic Society 2009 

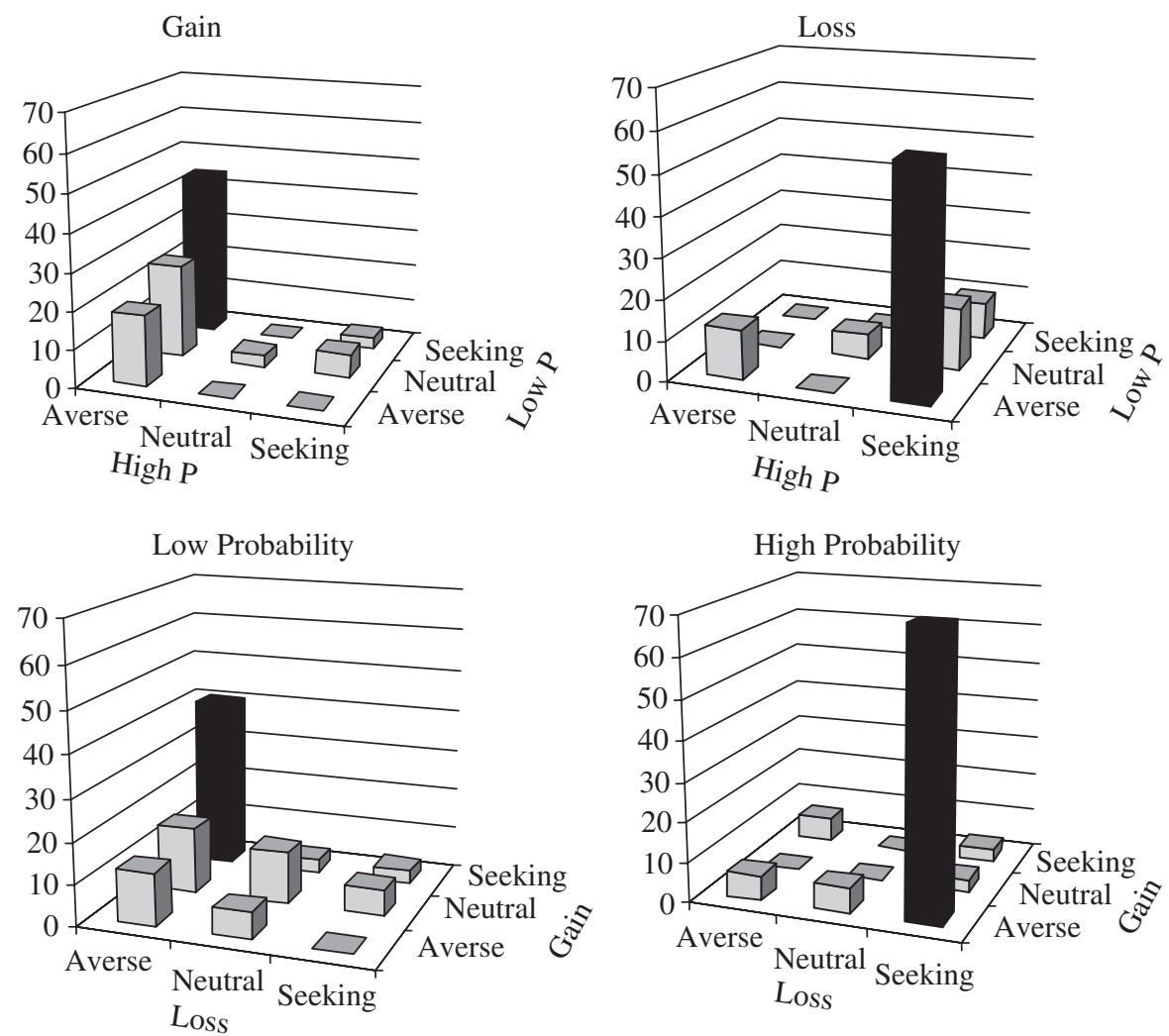

Fig. 2. Risk Attitudes of Price-participants in the Price Task

Note. 32 participants, first-round decisions, percentages on vertical axis. The proportion with the predicted reflection between high and low-probability prospects is $44 \%$ for gains and $56 \%$ for losses. For low-probability prospects $41 \%$ reflect as predicted between losses and gains and for high-probability prospects $56 \%$ exhibit the reflection.

the remaining participants reflect in the predicted manner. ${ }^{19}$ Thus using the price elicitation there is substantial support for the fourfold pattern, whether we focus on reflection of risk attitudes between gains and losses, or between low and highprobability prospects.

While Figure 2 allows us to look at the two-way reflection it is also of interest to determine whether the fraction of those who exhibit the entire fourfold pattern over the four prospects exceeds the fraction expected if people were equally likely to have any combination of risk attitudes. Looking only at first-round prices, we find that 10 of 32 price-participants, or $34 \%$, report prices that are fully consistent with the fourfold pattern. At most 4 participants choose any of the other combinations of risk attitudes. Since there are 3 possible risk attitudes for 4 prospects, there are 81 possible combinations. Ignoring individuals with risk-neutral decisions there are 16 possible

\footnotetext{
19 p-values are at 0.002 or lower. A stronger test of the fourfold pattern is whether the majority of participants reflect as predicted or whether all participants have the predicted reflection. Throughout the article we focus on the weaker test.
}

(C) The Author(s). Journal compilation (c) Royal Economic Society 2009 
combinations. With p-values of less than 0.001 we reject the null that the proportion of all participants choosing the fourfold pattern at most equals $1 / 81$, as well as the hypothesis that at most $1 / 16$ of the participants who are never risk-neutral exhibit the fourfold pattern.

As a control for error, participants were given two opportunities to review and change their decisions. Most people chose to revise their decisions. Of 96 participants only 19 never changed any of their decisions between the first and third round. Recall that second-round decisions are made after all six prospects in a task are reviewed and the third-round decision is made after the participant has completed both tasks and reviewed the decisions of all 12 prospects. The elicited risk attitudes over the three rounds are reported for the price-participants in Table 3(a). The attitudes predicted by the fourfold pattern are italicised. Although revisions slightly diminish the support for the fourfold pattern it remains across the three rounds. For every round we can reject the null that $1 / 3$ or fewer of the price-participants choose prices consistent with the fourfold pattern. For the low-probability loss and for the two high-probability prospects we reject the null with p-values less than 0.01 , for the low-probability gain prospect the p-value is instead 0.08 in the first round and less than 0.05 in the second and third round. Thus the fourfold pattern remains despite revisions.

To study the robustness of the fourfold pattern further under the price elicitation we also examine the prices that result when participants first have used the choice task and then evaluate the same prospects using the price task. As seen in Table 3(b), for these choice-participants the mean prices are also consistent with the fourfold pattern. ${ }^{20}$ When we characterise each individual's risk attitude according to their reported price,

Table 3

Risk Attitudes by Prospect and Round

(a) Price-participants in Price Task, $n=32$

(b) Choice-participants in Price Task, $n=64$

\begin{tabular}{|c|c|c|c|c|c|c|c|c|c|c|c|c|}
\hline \multirow[b]{4}{*}{ Risk attitude: } & \multicolumn{6}{|c|}{ Low Probability $(p=0.1)$} & \multicolumn{6}{|c|}{ High Probability $(\mathrm{p}=0.8)$} \\
\hline & \multirow{2}{*}{\multicolumn{3}{|c|}{$\frac{\text { Gain }(+\$ 20)}{\text { Round }}$}} & \multirow{2}{*}{\multicolumn{3}{|c|}{$\frac{\text { Loss }(-\$ 20)}{\text { Round }}$}} & \multirow{2}{*}{\multicolumn{3}{|c|}{$\frac{\text { Gain }(+\$ 20)}{\text { Round }}$}} & \multirow{2}{*}{\multicolumn{3}{|c|}{$\frac{\text { Loss }(-\$ 20)}{\text { Round }}$}} \\
\hline & & & & & & & & & & & & \\
\hline & 1 & 2 & 3 & 1 & 2 & 3 & 1 & 2 & 3 & 1 & 2 & 3 \\
\hline \multicolumn{13}{|l|}{ (a) } \\
\hline Averse & 19 & 16 & 16 & 69 & 66 & 63 & 88 & 78 & 75 & 13 & 22 & 22 \\
\hline Neutral & 34 & 34 & 34 & 22 & 25 & 25 & 3 & 6 & 9 & 6 & 6 & 6 \\
\hline Seeking & 47 & 50 & 50 & 9 & 9 & 13 & 9 & 16 & 16 & 81 & 72 & 72 \\
\hline \multicolumn{13}{|l|}{ (b) } \\
\hline Averse & 27 & 25 & 25 & 56 & 52 & 52 & 77 & 75 & 75 & 11 & 9 & 9 \\
\hline Neutral & 34 & 36 & 36 & 22 & 23 & 25 & 8 & 11 & 11 & 11 & 11 & 11 \\
\hline Seeking & 39 & 39 & 39 & 22 & 25 & 23 & 16 & 14 & 14 & 78 & 80 & 80 \\
\hline
\end{tabular}

Notes. Percentages in cells. Italicised cells show the fourfold pattern predicitions.

${ }^{20}$ For the gain prospects with $10 \%, 40 \%$ and $80 \%$ chance of winning the reported mean prices are $\$ 3.1$, $\$ 6.9$ and $\$ 12.0$. For the three loss prospects the mean prices are $-\$ 4.5,-\$ 7.8$ and $-\$ 12.4$, respectively. When replicating Figure 2 for the choice-participants the modal choice continues to be that predicted.

(C) The Author(s). Journal compilation (C) Royal Economic Society 2009 
Table 4

Choice-participants in the Choice Task

\begin{tabular}{llcccc}
\hline \hline Prospect & & $\begin{array}{c}\text { Expected } \\
\text { Value }\end{array}$ & $\begin{array}{c}\text { Percentage } \\
\text { Choosing Gamble }\end{array}$ & $\begin{array}{c}\text { p-value for } \\
\text { Exact Test }\end{array}$ & $\begin{array}{c}\text { Median } \\
\text { Risk Attitude }\end{array}$ \\
\hline Gain $+\$ 20$ & $1 \cdot \mathrm{p}=0.1$ & $+\$ 2$ & 50.0 & 1.000 & $\begin{array}{c}\text { Neutral } \\
\text { Loss }-\$ 20\end{array}$ \\
& 2. $\mathrm{p}=0.4$ & $+\$ 8$ & 39.1 & 0.103 & Averse \\
& 3. $\mathrm{p}=0.8$ & $+\$ 16$ & 56.3 & 0.382 & Seeking \\
& 4. $\mathrm{p}=0.1$ & $-\$ 2$ & 68.8 & 0.004 & Seeking \\
& 5. $\mathrm{p}=0.4$ & $-\$ 8$ & 56.3 & 0.382 & Seeking \\
& 6. $\mathrm{p}=0.8$ & $-\$ 16$ & 40.6 & 0.169 & Averse \\
\hline \hline
\end{tabular}

Notes. 64 participants, first-round decisions. The test is an exact binomial test of the null hypothesis that the proportion choosing the gamble $=0.5$.

the distribution of individuals is consistent with the fourfold pattern for all but the low-probability gain. While the p-value for the null is 0.2 for the low-probability gain it is less than 0.01 for the three other prospects. Thus independent of order we find that risk attitudes elicited with the price task are consistent with the fourfold pattern.

\section{Risk Attitudes from Choice Elicitations}

While participants in the price task were asked to report a monetary equivalent for each of the six prospects, in the choice task participants only needed to decide whether they preferred the prospect or its expected value. Despite the prospects being the same across the two elicitations, we do find very different results. We start by examining the initial choices by participants who only had been presented with the choice task. Table 4 shows the proportion of choice-participants who chose the gamble over its expected value and the implied median risk attitude for the first round choices. Assuming that indifference causes individuals to randomise, the implied risk attitudes tend to be statistically indistinguishable from risk-neutrality and, if anything, they are opposite of that predicted by the fourfold pattern.

The same result appears when we look at within-participant reflections in Figure 3. The first panel examines the reflection of risk attitudes between high and lowprobability prospects conditional on the prospect being a gain or a loss, and the second panel illustrates reflection when changing a loss to a gain conditional on it being a low or high-probability prospect. The highlighted cells illustrate reflections consistent with the fourfold pattern.

The first noticeable difference from Figure 2 is that the distribution of risk attitudes is less extreme and that a much smaller fraction of individuals exhibit the reflection predicted by the fourfold pattern. In fact, in three of the four cases the cell predicted by the fourfold pattern is observed with the lowest frequency. Statistical tests of the reflections confirm what one would expect from the patterns in Figure 3. In none of the four cases can we reject the null hypothesis that at most $25 \%$ of participants make the predicted choices in favour of the fourfold pattern prediction: all p-values exceed 0.75 . While risk attitudes do reflect between gains and losses and low and high probabilities - reflections are the modal outcome in each of the 4 cases - the pattern tends 

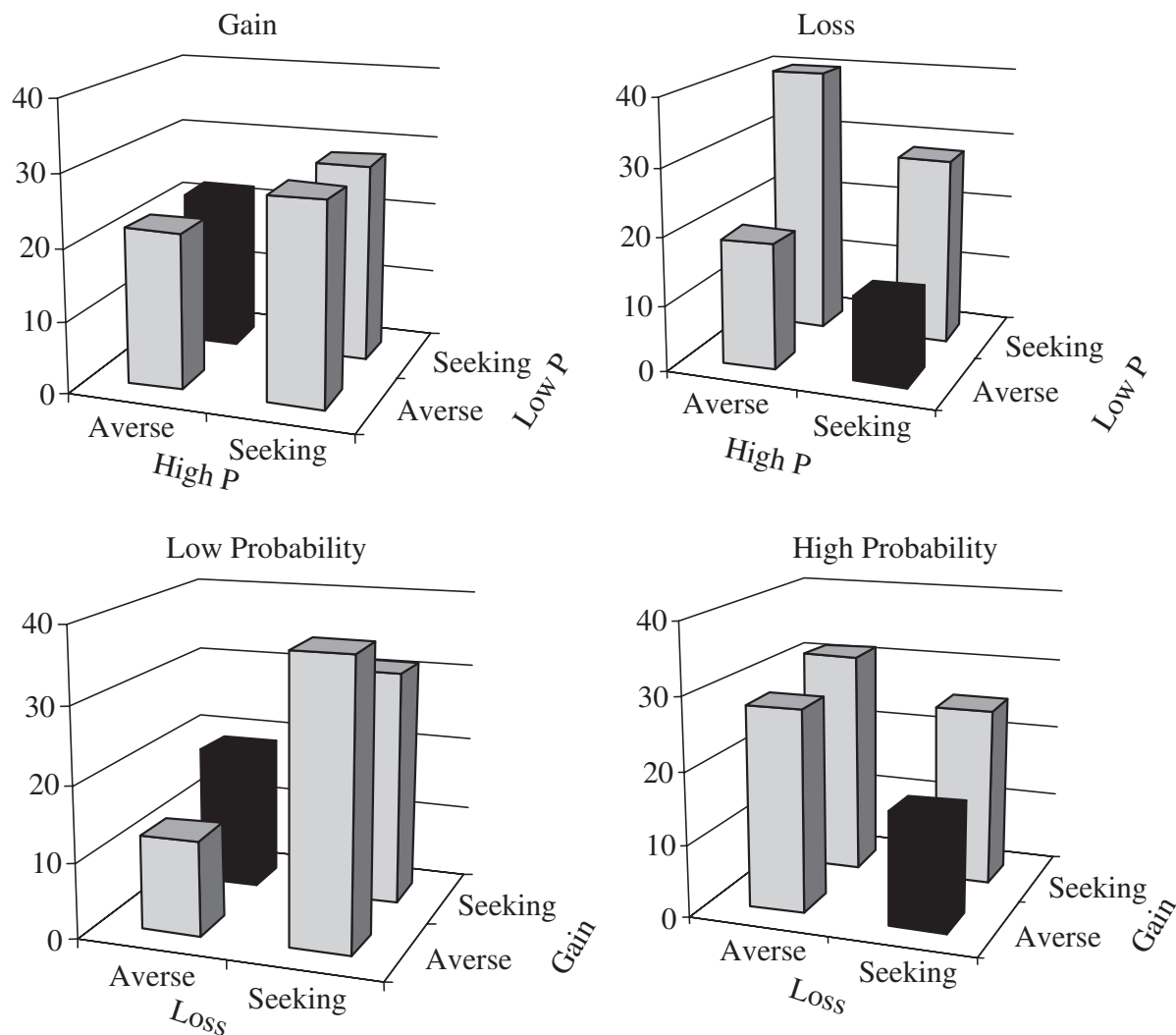

Fig. 3. Risk Attitudes of Choice-participants in the Choice Task

Note. 64 participants, first-round decisions, percentages on vertical axis. The proportion with the expected reflection between high and low-probability prospects is $22 \%$ for gains and $13 \%$ for losses. For low-probability prospects $19 \%$ exhibit the predicted reflection between losses and gains, the comparable number of high-probability prospects is $16 \%$.

to be the opposite of the fourfold pattern. With the exception of the gain prospects, we can reject the hypothesis that, of the people who reflect risk attitudes, at least half reflect in the predicted manner. ${ }^{21}$ The preferences elicited with the choice task also provide limited evidence for the entire fourfold pattern. Only 4 of 64 participants make choices consistent with the full fourfold pattern, precisely the proportion we would expect if the fourfold pattern had no predictive power. ${ }^{22}$

As with the price task the opportunity to revise decisions in the choice task does not result in much change in the elicited risk attitudes. Table $5(a)$ presents the attitudes for the three rounds of decisions for the choice-participants. We cannot reject the hypothesis that at most half the participants make choices consistent with the fourfold pattern in any of the twelve cases. Most p-values are above 0.80 and the lowest is 0.13 .

${ }^{21} \mathrm{p}=0.298$ when prospects are gains, whereas the $\mathrm{p}$-value is below 0.050 in the three other cases.

${ }^{22}$ With $\mathrm{p}=0.573$ we cannot reject the null that at most $1 / 16$ choose the predicted pattern. Note also that 7 participants make choices that are exactly opposite the fourfold pattern, and 10 participants pick the expected value only for prospect 6 .

(C) The Author(s). Journal compilation (C) Royal Economic Society 2009 
Table 5

Risk Attitudes by Prospect and Round

(a) Choice-participants in the Choice Task, $n=64$

(b) Price-participants in the Choice Task, $n=32$

\begin{tabular}{|c|c|c|c|c|c|c|c|c|c|c|c|c|}
\hline \multirow[b]{4}{*}{ Risk attitude: } & \multicolumn{6}{|c|}{ Low Probability $(\mathrm{p}=0.1)$} & \multicolumn{6}{|c|}{ High Probability $(\mathrm{p}=0.8)$} \\
\hline & \multirow{2}{*}{\multicolumn{3}{|c|}{$\frac{\text { Gain }(+\$ 20)}{\text { Round }}$}} & \multirow{2}{*}{\multicolumn{3}{|c|}{$\frac{\text { Loss }(-\$ 20)}{\text { Round }}$}} & \multirow{2}{*}{\multicolumn{3}{|c|}{$\frac{\text { Gain }(+\$ 20)}{\text { Round }}$}} & \multirow{2}{*}{\multicolumn{3}{|c|}{$\begin{array}{c}\text { Loss }(-\$ 20) \\
\text { Round }\end{array}$}} \\
\hline & & & & & & & & & & & & \\
\hline & 1 & 2 & 3 & 1 & 2 & 3 & 1 & 2 & 3 & 1 & 2 & 3 \\
\hline \multicolumn{13}{|l|}{ (a) } \\
\hline Averse & 50 & 44 & 42 & 31 & 36 & 34 & 44 & 45 & 45 & 59 & 56 & 58 \\
\hline Seeking & 50 & 56 & 58 & 69 & 64 & 66 & 56 & 55 & 55 & 41 & 44 & 42 \\
\hline \multicolumn{13}{|l|}{ (b) } \\
\hline Averse & 50 & 41 & 44 & 44 & 38 & 41 & 41 & 41 & 41 & 72 & 69 & 69 \\
\hline Seeking & 50 & 59 & 56 & 56 & 63 & 59 & 59 & 59 & 59 & 28 & 31 & 31 \\
\hline
\end{tabular}

Notes. Percentages in cells. Italicised cells show the fourfold pattern predictions.

The results are similar for the price-participants in Table $5(b)$. All p-values are above 0.80 except for low-probability gains and the lowest of those is 0.19 . Thus our finding that the fourfold pattern does not arise in the choice-based procedure is robust to ordering and to the expected wealth effect from participation in the first task. It is particularly striking that the results remain in the third round when participants simultaneously review their price and choice decisions. While our results show strong support for the fourfold pattern in the price task, this is not the case in the choice task.

\section{Discussion and Conclusion}

Presenting participants with a few simple gambles we find that the fourfold pattern of risk attitudes is sensitive to the preference elicitation mechanism. While the fourfold pattern accurately characterises people's pricing decisions, it does no better than chance at predicting their choices between gambles and the corresponding expected value. These results hold regardless of whether we start with the price or the choice task and are robust to simultaneously reviewing decisions under the two tasks.

Our study raises the question of why the elicited risk attitudes are consistent with the fourfold pattern under the price task but not under the choice task. One possible explanation is that the transparency varies between the two elicitation mechanisms. Making a choice between a lottery and a sure outcome is a simple and familiar task, while the pricing method used here and in previous experiments is more complicated. Individuals have limited experience pricing objects and may find it particularly difficult to price a gamble. Perhaps the inexperience causes them to adopt rules of thumb that generate the fourfold pattern of risk attitudes. For example, participants may pick a naïve rule whereby the price is selected about halfway between the best and the worst outcomes of the gamble but moved a bit towards the more likely outcome. While the price rule may be the same for gains and losses, the risk attitudes implied by these prices would be the reverse of one another. For example, if a $10 \%$ chance of $\$ 20$ is assessed 
at $\$ 5$ in the gain domain and $-\$ 5$ in the loss domain, then the individual is said to be risk seeking over gains and risk-averse over losses. Thus naïve pricing rules could give rise to the fourfold pattern. It may be argued that the similarity in the absolute value of the reported prices for losses and gains in Table 2 is consistent with similar pricing rules in the two domains. The BDM procedure used to secure that the price elicitation is incentive compatible may be another reason why the fourfold pattern arises in the price task. Even if well understood this procedure may bias the reported prices in favour of the fourfold pattern. Specifically, the bounds on the distribution of the randomly determined prices may truncate the reported willingness to pay for gambles of low expected value from the left, while those of high expected value are truncated from the right. Such a truncation can give rise to the fourfold pattern. Finally, the greater complexity of the BDM procedure may in and of itself cause the elicited preferences to differ between the two methods. As argued by the literature on dual selves, we may find greater behavioural anomalies when the cognitive load is high, because in such cases the restraint on the impulsive self is low. Thus the support of the fourfold pattern in the price task may be due to the cognitive load being greater than in the choice task.

Another reason why the price and choice-based procedures elicit different preferences may be that they are less similar than they initially appear. While the evaluated prospects are the same, the possible payoffs vary between the two procedures. In addition to the randomly generated BDM price differing from the prospect's expected value, the potential outcomes of the price and choice task are rather different when evaluating gain prospects. In the price task participants are asked to pay for the gain gamble, whereas participants in the choice task are asked to choose between the gamble and its expected value. ${ }^{23}$ Thus expected wealth is higher in the choice task and the prospect is solely in the gain domain. A participant either chooses the positive expected value or faces two possible outcomes: a gain of $\$ 20$ or a gain of $\$ 0$. In contrast it can be argued that the prospect in the price task is mixed over losses and gains. Specifically participants may end up paying for a gamble that does not win any money, thereby losing the BDM-generated price. The anticipation of such a loss may cause the elicited price to be influenced by loss aversion. ${ }^{24}$

While we cannot adjust for the differences between paying a randomly determined price versus the expected value, it is possible to make the price and choice procedure more similar in the gain domain. We conducted an additional treatment to examine if our results were sensitive to such a modification. Since the objective of the article is to examine the support for, and procedural invariance of, the fourfold pattern of risk attitudes and since the price task clearly demonstrates this pattern, the choice task was revised to be more comparable to the price task. Specifically, participants were asked to choose whether they would give up the gamble's expected value in return for the gamble. In addition to modifying the choice task in the gain domain, we also expanded the participant's choice set

\footnotetext{
23 This type of inconsistency is also present in previous comparisons between price and choice elicitations, see e.g. Slovic and Lichtenstein (1968).

24 It may be argued that using a price task inherently results in mixed prospects. If participants instead were asked to state the amount they are willing to accept then a similar situation will arise over losses. Some participants would receive payments in return for accepting a negative prospect and then not lose any money. Changing the price task to be similar to that of the choice task would require that we framed the price task in terms of willingness to pay in the loss domain and willingness to accept in the gain domain.
}

(C) The Author(s). Journal compilation (C) Royal Economic Society 2009 
to include an option of indifference. ${ }^{25}$ That is, the participants could choose the prospect, its expected value, or a 'don't care' option, where the flip of a coin determines whether they receive the expected value of the prospect or play the prospect.

A total of 32 new participants, from the same subject pool, participated in the new treatment. Participants were first given the new-choice task and then the original price task. Our results show, first, that very few participants select the 'don't care' option. ${ }^{26}$ Second, our earlier finding is robust. With the exception of the low-probability gain the implied risk attitudes for the majority of participants in the choice task are the opposite of that predicted by the fourfold pattern. Furthermore, the reflections of risk attitudes are not consistent with the prediction. ${ }^{27}$ In none of the four examined reflections cases can we reject the hypothesis that the proportion reflecting according to the fourfold pattern is no larger than what we would expect from random choice. ${ }^{28}$ With the exception of the low probability gain, the modal choices tend to be the exact opposite of the fourfold pattern prediction. In the three other cases, we reject the hypothesis that at least $50 \%$ of those who reflect risk attitudes do so in the predicted direction, with p-values below 0.004 . Over the four relevant prospects none of the 32 participants made choices that were consistent with the full fourfold pattern. In fact the modal pattern was the exact opposite of the fourfold pattern, with 5 participants choosing this combination. After evaluating the six gambles with the new-choice task, participants were asked to evaluate the gambles using the price task. Examining these decisions we once again find that the risk attitudes derived with the price procedure are consistent with the fourfold pattern. Thus despite the greater similarity in the two procedures we continue to find evidence of the fourfold pattern in the price task but not in the choice task.

Much like Slovic and Lichtenstein (1968) our results demonstrate that the price ordering of prospects can be very different from the choice ordering. ${ }^{29}$ Looking only at third-round decisions we see that of the participants who were either risk-averse or risk-seeking in the price task, $42 \%$ had the opposite risk attitude when asked to evaluate the same gamble with the new-choice task. ${ }^{30}$ If the majority of participants have one risk

25 In the price task participants indicate risk-neutrality by reporting that they are willing to pay the gamble's expected value to play the gamble.

${ }^{26}$ For each prospect, an average of $14.5 \%$ are indifferent.

27 The proportion with the predicted reflection between high and low-probability prospects is $16 \%$ for gains and $6 \%$ for losses. For low-probability prospects $16 \%$ reflect as predicted between losses and gains, and for high-probability prospects $6 \%$ exhibit the reflection.

28 That is, we cannot reject that at most $1 / 9$ of all participants reflect as predicted, nor can we reject that at most $1 / 4$ of the participants who never are risk-neutral exhibit the predicted reflection. The smallest p-value is 0.273 .

29 Their example involved two lotteries, one with a high probability of winning a small amount and the other with a low probability of winning a large amount but with equal expected values. They showed that most participants choose the high-probability lottery over the low-probability one but priced the low-probability lottery higher than the high-probability lottery; see Grether and Plott (1979) for a careful replication of these results. To explain this preference reversal they argue that when making a choice people focus on the probability of the prospects but when determining a price they focus on the payoffs. It is not clear how one would apply this explanation to the present scenario.

30 Tversky and Kahneman (1992) proposed that reversals of the Slovic-Lichtenstein type are caused by a tendency to overprice prospects. Thus, in the choice task participants should appear more risk-averse over gains. Since the predominant risk attitude in the choice task tends to be the opposite of that in the price task, the preference reversals between the two methods can not be explained by a systematic overpricing of prospects. Looking at third-round results over gains we find that $47 \%$ of the participants who were risk-averse in the price task become risk-seeking in the choice task, whereas only $36 \%$ of those who were risk-seeking in the price task become risk-averse in the choice task.

(C) The Author(s). Journal compilation (C) Royal Economic Society 2009 
attitude in the price task then the majority of participants tend to have the opposite risk attitude in the choice task. For example, in the high-probability loss prospect, 3/4 of participants are willing to pay less than the gamble's expected value to avoid the risky loss, yet half of these same participants choose the certain loss when given the choice between the gamble and a certain loss of the expected value.

The consequences of procedural variance in risk attitudes are substantial. Not only does it raise the serious question of determining which procedure is appropriate when eliciting risk attitudes but it may also have important implications for how we choose to present risky outcomes. Consider for example a person purchasing a new car. She may have a choice between a car with a particular safety feature that will protect against a low probability of a large loss and a car that does not have that feature. If this is perceived as a choice task, the car without the safety feature may be chosen. However, if the salesperson frames the decision as a feature available at an additional cost, it becomes a price task. The buyer may then approach the problem with a risk-averse attitude and buy the safety-equipped car.

\section{University of Oregon and NBER}

University of New Mexico

University of Pittsburgh

Submitted: 28 August 2007

Accepted: 3 March 2009

\section{References}

Battalio, R., Kagel, J. and Jiranyakul, K. (1990). 'Testing between alternative models of choice under uncertainty: some initial results', Journal of Risk and Uncertainty, vol. 3, pp. 25-50.

Benjamin, D.J., Brown, S.A. and Shapiro, J.M. (2006). "Who is "behavioral"? Cognitive ability and anomalous preferences', Mimeo: Harvard University.

Bernheim, D and Rangel, A. (2004). 'Addiction and cue-triggered decision processes', American Economic Review, vol. 94(5), pp. 1558-90.

Brocas, I. and Carrillo, J. (2005). 'The brain as a hierarchical organization'. CEPR Discussion Paper No. 5168, (August).

Camerer, C. (1989). 'An experimental test of several generalized utility theories', Journal of Risk and Uncertainty, vol. 2, pp. 61-104.

Camerer, C. (1998). 'Bounded rationality in individual decision making', Experimental Economics, vol. 1(2), pp. $163-83$.

Camerer, C. (2000). 'Prospect theory in the wild: evidence from the field'. in (D. Kahneman and A. Tversky, eds.), Choices, Values, and Frames, pp. 288-300, New York: Cambridge University Press.

Camerer, C. and Ho, T-H. (1994). 'Violations of the betweenness axiom and nonlinearity in probabilities', Journal of Risk and Uncertainty, vol. 8, pp. 167-96.

Camerer, C. and Hogarth, R. (1999). 'The effects of financial incentives in economics experiments: a review and capital-labor-production framework', Journal of Risk and Uncertainty, vol. 19, pp. 7-42.

Fudenberg, D. and Levine, D. (2006). 'A dual self model of impulse control', American Economic Review, vol. 96(5), pp. 1449-76.

Gonzalez, R. and Wu, G. (1999). 'On the shape of the probability weighting function', Cognitive Psychology, vol. 38(1), pp. 129-66.

Grether, D.M. and Plott, C.R. (1979). 'Economic theory of choice and preference reversal phenomenon', American Economic Review, vol. 69(4), pp. 623-38.

Harbaugh, W.T., Krause, K. and Vesterlund, L. (2002). 'Risk attitudes of children and adults: choices over small and large probability gains and losses', Experimental Economics, vol. 5(1), pp. 53-84.

Harless, D. and Camerer, C. (1994). 'The predictive utility of generalized expected utility theories', Econometrica, vol. 62(6), pp. 1251-90.

Hertwig, R., Barron, G., Weber, E.U. and Erev, I. (2004). 'Decisions from experience and the effect of rare events in risky choice', Psychological Science, vol. 15(8), pp. 534-9.

(C) The Author(s). Journal compilation (C) Royal Economic Society 2009 
Hey, J.D. and Orme, C. (1994). 'Investigating generalizations of expected utility theory using experimental data', Econometrica, vol. 62(6), pp. 1291-326.

Holt, C.A. and Laury, S.K. (2002). 'Risk aversion and incentive effects', American Economic Review, vol. 92(5), pp. 1644-55.

Kachelmeier, S.J., and Shehata, M. (1992). 'Examining risk preferences under high monetary incentives: experimental evidence from the People's Republic of China', American Economic Review, vol. 82(5), pp. 1120-41.

Kahneman, D. and Tversky, A. (1979). 'Prospect theory: an analysis of decision under risk', Econometrica, vol. $47(2)$, pp. 263-91.

Laury, S.K. (2002). 'Pay one or pay all: random selection of one choice for payment'. Andrew Young School of Policy Studies Research Paper Series No. 06-13.

Laury, S.K., and Holt, C.A. (2008). 'Further reflections on prospect theory', in (J.C. Cox and G. Harrison, eds.), Risk Aversion in Experiments (Experimental Economics, Volume 12), pp. 405-40, Greenwich, CT: JAI Press.

Loewenstein, G. and O'Donoghue, T. (2005). 'Animal spirits: affective and deliberative processes in economic behavior', Working paper, Cornell University.

Machina, M. (1987). 'Choice under uncertainty: problems solved and unsolved', Journal of Economic Perspectives, vol. 1, pp. 121-54.

Ozdenoren, E., Salant, S. and Silverman, D. (2006). 'Willpower and the optimal control of visceral urges', NBER Working Paper No W122278.

Prelec, D. (1998). 'The probability weighting function', Econometrica, vol. 66(3), pp. 497-527.

Schmidt, U., Starmer, C. and Sugden, R. (2005). 'Explaining preference reversal with third-generation prospect theory', CeDEx Discussion Paper No. 2005-19, available at http://www.nottingham.ac.uk/ economics/cedex/papers/2005-19.pdf

Schoemaker, P. (1982). 'The expected utility model: its variants, purposes, evidence and limitations'. Journal of Economic Literature, vol. 20, pp. 529-63.

Slovic, P. and Lichtenstein, S. (1968). 'Relative importance of probabilities and payoffs in risk taking', Journal of Experimental Psychology Monograph, vol. 78, pp. 1-18.

Starmer, C. (2000). 'Developments in non-expected utility theory: the hunt for a descriptive theory of choice under risk', Journal of Economic Literature, vol. 38, pp. 332-82.

Starmer, C., Sugden, R. and Cubitt, R. (1998). 'On the validity of the random lottery incentive system', Experimental Economics, vol. 1, pp. 115-31.

Tversky, A. and Fox, C. (1995). 'Weighing risk and uncertainty', Psychological Review, vol. 102, pp. $269-83$.

Tversky, A., and Kahneman, D. (1986). 'Rational choice and the framing of decisions', Journal of Business, vol. 59 , pp. S251-78.

Tversky, A. and Kahneman, D. (1992). 'Advances in prospect theory: cumulative representation of uncertainty', Journal of Risk and Uncertainty, vol. 5, pp. 297-323.

Wu, G. and Gonzalez, R. (1996). 'Curvature of the probability weighting function', Management Science, vol. 42, pp. 1676-90. 